\title{
Análise tomográfica dos parâmetros anatômicos para inserção dos parafusos de trajeto cortical*
}

\section{Tomographic Analysis of the Anatomical Parameters for the Insertion of Cortical Bone Screws}

\author{
Carlos Fernando Pereira Silva Herrero ${ }^{1}$ Rafael Campos Fróes Marangoni ${ }^{10}$ \\ ${ }^{1}$ Departamento de Ortopedia e Anestesiologia, Faculdade de \\ Medicina de Ribeirão Preto, Universidade de São Paulo, Ribeirão \\ Endereço para correspondência Rafael Campos Fróes Marangoni, \\ Preto, São Paulo, SP, Brasil \\ MD, rua Padre Diogo Feijó, 101, Taubaté, SP, Brasil, CEP 12030-160 \\ (e-mail: rafaelcfmarangoni@gmail.com).
}

Rev Bras Ortop 2022;57(1):47-54.

\begin{abstract}
Resumo
Objetivo Estudar os parâmetros anatômicos do trajeto de inserção do parafuso cortical e descrever sua técnica.

Métodos Analisaram-se exames de tomografia computadorizada de 30 pacientes, e as medidas nas vértebras de L1 a L5 bilateralmente. Um segundo observador avaliou dez exames aleatoriamente. Os parâmetros incluíram o ângulo lateral ( $\mathrm{AL}$ ) e o diâmetro do parafuso (DP) como variáveis axiais, e o ângulo cranial (AC) e o comprimento do parafuso (CP) como variáveis sagitais.

Resultados No total, havia 15 pacientes do sexo masculino (média de idade de 31,33 anos) e 15 do sexo feminino (média de idade de 32,01 anos). O AL variou de $13,8^{\circ}$ a $20,89^{\circ}$, com uma tendência de aumento no sentido de proximal a distal. O AC variou de $17,5^{\circ}$ a $24,9^{\circ}$, com tendência de diminuição no sentido caudal. O DP variou de $2,3 \mathrm{~mm}$ a $7,2 \mathrm{~mm}$, havendo uma tendência ao aumento conforme avançamos de proximal a

Palavras-chave

- coluna vertebral

- procedimentos cirúrgicos minimamente invasivos

- parafusos ósseos distal. O CP variou de $19 \mathrm{~mm}$ a $45 \mathrm{~mm}$, havendo uma tendência de diminuição conforme avançamos de proximal (L1) a distal (L5). Não houve diferença estatística entre os sexos, nem diferenças na confiabilidade interobservador, quanto aos valores estudados quando comparados os lados.

Conclusão A trajetória do parafuso de trajeto cortical apresenta variações em diferentes populações. Assim, recomendamos o estudo pré-operatório de imagens para reduzir os riscos cirúrgicos relacionados à técnica.
\end{abstract}

Trabalho desenvolvido no Hospital das Clínicas de Ribeirão Preto, Faculdade de Medicina de Ribeirão Preto, Universidade de São Paulo, Ribeirão Preto, São Paulo, SP, Brasil.

\section{Recebido:}

27 de julho de 2020 ,

Aceito após revisão:

11 de fevereiro de 2021 ,
DOI https://doi.org/ 10.1055/s-0041-1731672. ISSN 0102-3616. (c) 2022. Sociedade Brasileira de Ortopedia e Traumatologia. All rights reserved.

This is an open access article published by Thieme under the terms of the Creative Commons Attribution-NonDerivative-NonCommercial-License, permitting copying and reproduction so long as the original work is given appropriate credit. Contents may not be used for commercial purposes, or adapted, remixed, transformed or built upon. (https://creativecommons.org/ licenses/by-nc-nd/4.0/)

Thieme Revinter Publicações Ltda., Rua do Matoso 170, Rio de Janeiro, RJ, CEP 20270-135, Brazil 


\begin{abstract}
Keywords

- spine

- minimally invasive surgical procedures

- bone screws

Objective To study the parameters related to the insertion path of cortical screws and to describe this technique.

Methods Computed tomography (CT) scans of 30 patients, as well as the measurements from the $\mathrm{L} 1$ to the $\mathrm{L} 5$ vertebrae, were studied. A second observer evaluated ten randomly-selected exams. The parameters studied included the lateral angle (LA) and the screw diameter (SD) as axial variables, and the cranial angle (CA) and screw length (SL) as sagittal variables.

Results We studied 15 male patients (mean age: 31.33 years) and 15 female patients (mean age: 32.01 years). The LA varied between $13.8^{\circ}$ and $20.89^{\circ}$, with a tendency to increase in the proximal to distal direction. The CA varied from $17.5^{\circ}$ to $24.9^{\circ}$, with a tendency to decrease in the caudal direction. The SD ranged from $2.3 \mathrm{~mm}$ to $7.2 \mathrm{~mm}$, with a tendency to increase as we progressed from proximal to distal. The SL varied from $19 \mathrm{~mm}$ to $45 \mathrm{~mm}$, with a tendency to decrease as we proceeded from proximal (L1) to distal (L5). No statistical difference was observed between the genders or in the interobserver agreement regarding the values studied when comparing the sides.

Conclusion The path of insertion of the cortical screw shows a variation in different populations. Therefore, we recommend a preoperative imaging study to reduce the surgical risks related to the technique.
\end{abstract}

\section{Introdução}

A fixação posterior da coluna lombar é o tratamento de escolha para diversas patologias da coluna vertebral. Diversas ferramentas podem ser usadas no tratamento, incluindo os amarrilhos, os ganchos, os parafusos de trajeto pedicular (PTPs) e, mais recentemente, o parafuso de trajeto cortical (PTC). ${ }^{1,2}$

Atualmente, o método de fixação mais utilizado é feito com sistemas de parafusos pediculares, e tem como fundamento a ancoragem dos implantes no osso esponjoso dos pedículos e do corpo vertebral. Assim, esta técnica de fixação pode estar sujeita a falha, principalmente na presença osteoporose. $^{1,2}$

Em 2009, Santoni et al. ${ }^{17}$ apresentaram um trajeto de inserção de parafusos pediculares conhecido como parafuso de trajeto cortical (cortical bone trajectory). Nesta técnica, a inserção do parafuso segue o trajeto distal-proximal e a direção medial-lateral, o que aumenta o contato com o osso cortical no pedículo e no corpo vertebral comparado ao método tradicional de fixação com PTPs. ${ }^{3-6}$ Estudos biomecânicos demonstraram que a técnica que usa os PTCs alcança resultados equivalentes ou melhores do que método tradicional. $^{7-9}$

Apesar de a anatomia do trajeto do osso cortical ter sido documentada em populações europeias e asiáticas, ${ }^{10-12}$ seus parâmetros anatômicos podem variar entre populações diferentes. Nossa hipótese foi a de que os parâmetros morfométricos do parafuso de trajeto cortical na população brasileira eram diferentes dos parâmetros previamente relatados na literatura, e que as técnicas utilizadas podem levar a erros de interpretação. Assim, o objetivo deste estudo foi descrever uma técnica de mensuração do trajeto do PTC, conduzindo uma avaliação detalhada do ponto de entrada, do trajeto, e das dimensões do PTC por meio de tomografia computadorizada (TC) em uma amostra da população brasileira.

\section{Material e Métodos}

O protocolo do estudo foi submetido e aprovado pelo Comitê de Ética em Pesquisa do Hospital em questão. Foram incluídos no estudo 15 pacientes do sexo masculino (idade média: $31,33 \pm 8,5$ anos) e 15 do sexo feminino (idade média: $32,01 \pm 6,1$ anos). Foram avaliadas retrospectivamente imagens de TC de um banco de dados selecionadas de modo aleatório. Foram selecionados exames de pacientes entre 18 e 45 anos de idade, e os pacientes com histórico de cirurgia prévia, evidência de doença degenerativa avançada, lesões traumáticas, infecção ou doença neoplásica na coluna vertebral foram excluídos com base no laudo radiológico e revisão posterior pelo avaliador.

Todos os exames de TC haviam sido feitos no mesmo aparelho, Big Bore 16-slice (Philips Healthcare, Cleveland, OH, EUA), seguindo o protocolo padrão da instituição. Para cada vértebra, havia imagens de reconstrução axial, sagital e coronal com $1 \mathrm{~mm}$ de espessura. Foram selecionadas imagens desde a primeira vértebra lombar (L1) até a quinta vértebra lombar (L5).

Os parâmetros axiais foram o ângulo lateral (AL), formado entre o trajeto do parafuso e o plano sagital, e o máximo diâmetro do parafuso (DP), a maior distância entre duas retas paralelas ao trajeto que tangenciam as corticais do pedículo. Os parâmetros sagitais incluíram o ângulo cranial (AC), formado entre o trajeto do parafuso e a placa terminal superior, e o comprimento do parafuso (CP), a distância entre o ponto de entrada e o cortical lateral da vértebra. Os parâmetros lineares foram medidos em milímetros, e os angulares, em graus. 
Dois observadores realizaram as medidas de maneira independente utilizando o programa de análise de imagens OsiriX, (Pixmeo SARL, Bernex, Suíça). O primeiro realizou a avaliação dos exames de 30 pacientes, e o segundo, a avaliação dos exames de 10 pacientes selecionados aleatoriamente dentre os 30 primeiros para a estimativa da confiabilidade interobservador.

O frequente desafio de transformar dados diagnósticos de imagem em informações cirurgicamente úteis envolve a dificuldade de descrever estruturas tridimensionais, tal como o trajeto do parafuso cortical, em duas dimensões. Para obter dados clinicamente aplicáveis a partir de imagens de TC, a avaliação multiplanar (axial, sagital e coronal) foi utilizada de maneira simultânea para medir todos os parâmetros.

\section{Análise das Imagens}

A primeira etapa da avaliação do trajeto do parafuso cortical envolveu a realização do alinhamento do eixo horizontal (pontilhado fino) nas imagens sagital e coronal com a placa terminal superior, e do eixo vertical (linha contínua) na imagem axial com o eixo sagital central da vértebra. Após essa etapa, localizava-se o centro do pedículo e inseria-se um ponto de marcação fixo (ponto fixo 1: ponto preto) nos três planos (-Figura 1).

Para a determinação do ponto de entrada, utilizou-se a imagem no plano coronal, que corta o istmo dos pedículos, e os eixos foram deslocados, de modo que o eixo vertical (linha contínua) tocasse no córtex medial do pedículo, e o eixo horizontal (pontilhado fino) estivesse sobre o córtex inferior do pedículo. Foi marcado um ponto fixo na imagem axial, na projeção do eixo vertical (linha contínua) no córtex posterior da lâmina (-Figura 2; ponto fixo 2: ponto branco).

Após esta etapa, o centro do eixo foi reposicionado no ponto fixo 1, no centro do pedículo, e, na imagem coronal, o eixo horizontal (pontilhado fino) foi deslocado para o córtex inferior do pedículo (-Figura 3). Na imagem axial, o eixo foi então girado de forma que o eixo vertical (linha contínua)

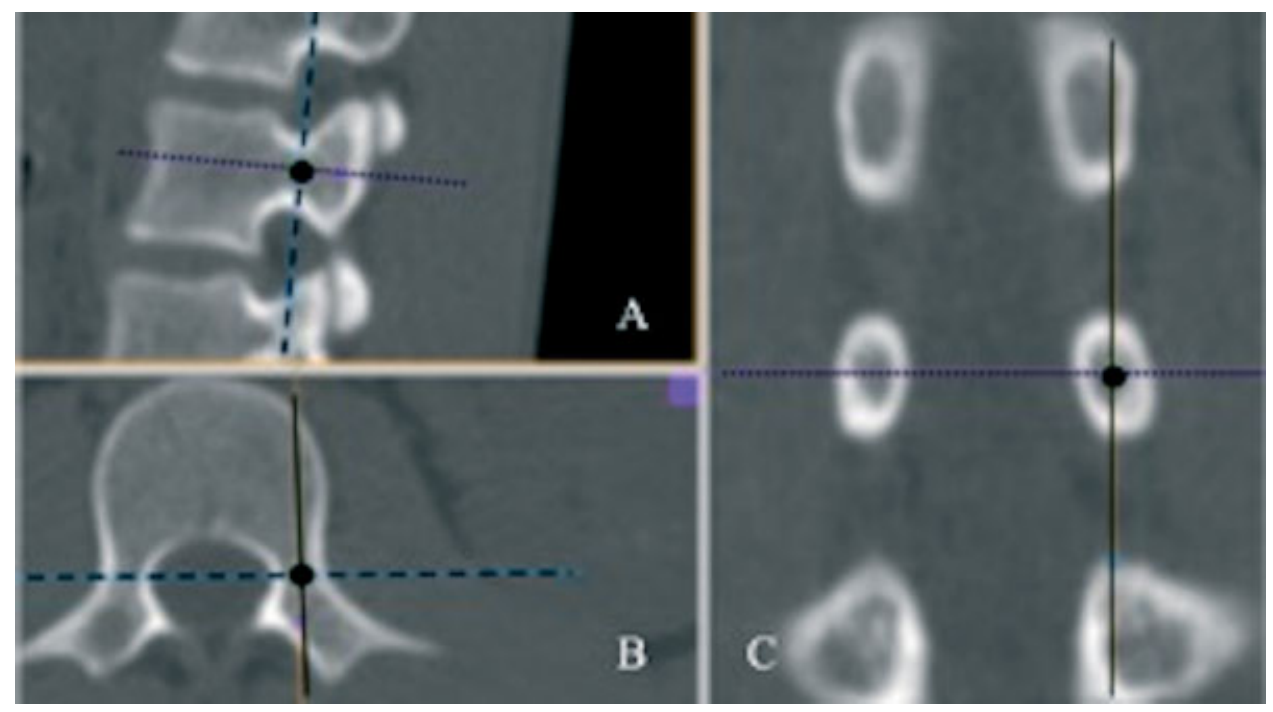

Fig. 1 Imagens sagital (A), axial (B) e coronal (C) de tomografia computadorizada evidenciando a demarcação do ponto fixo 1.

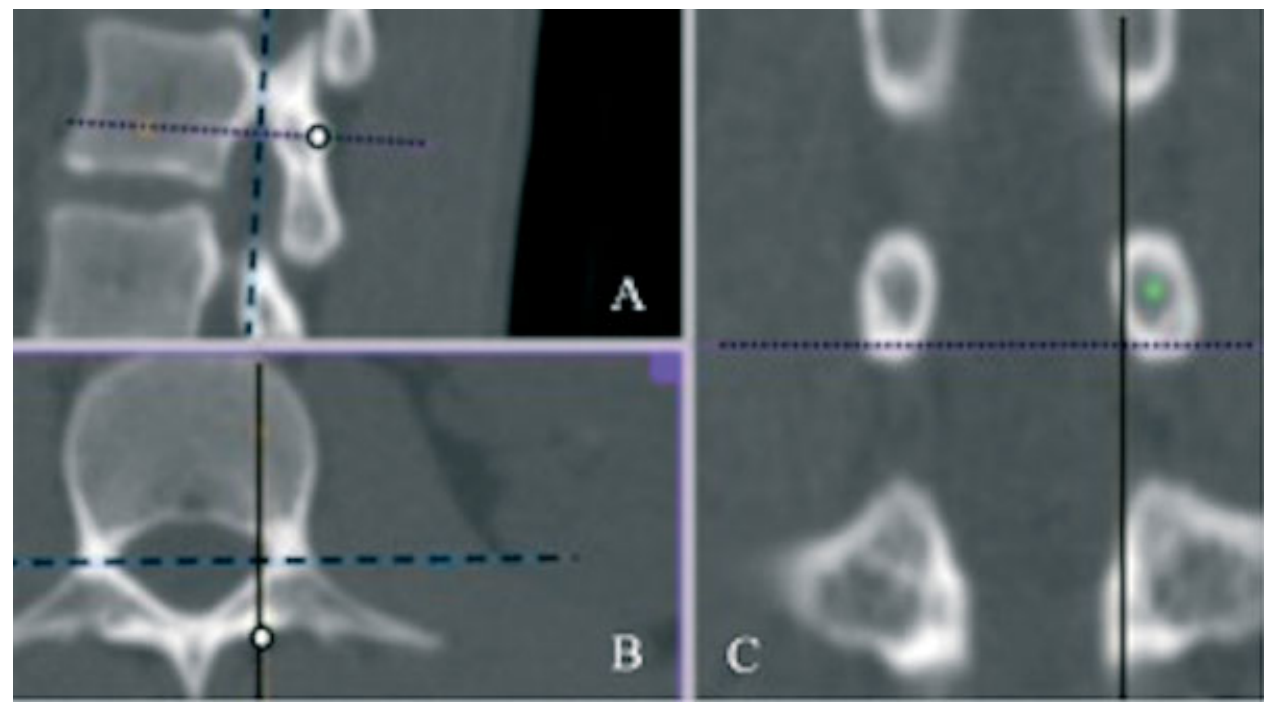

Fig. 2 Imagens sagital (A), axial (B) e coronal (C) de tomografia computadorizada evidenciando a demarcação do ponto fixo 2. 


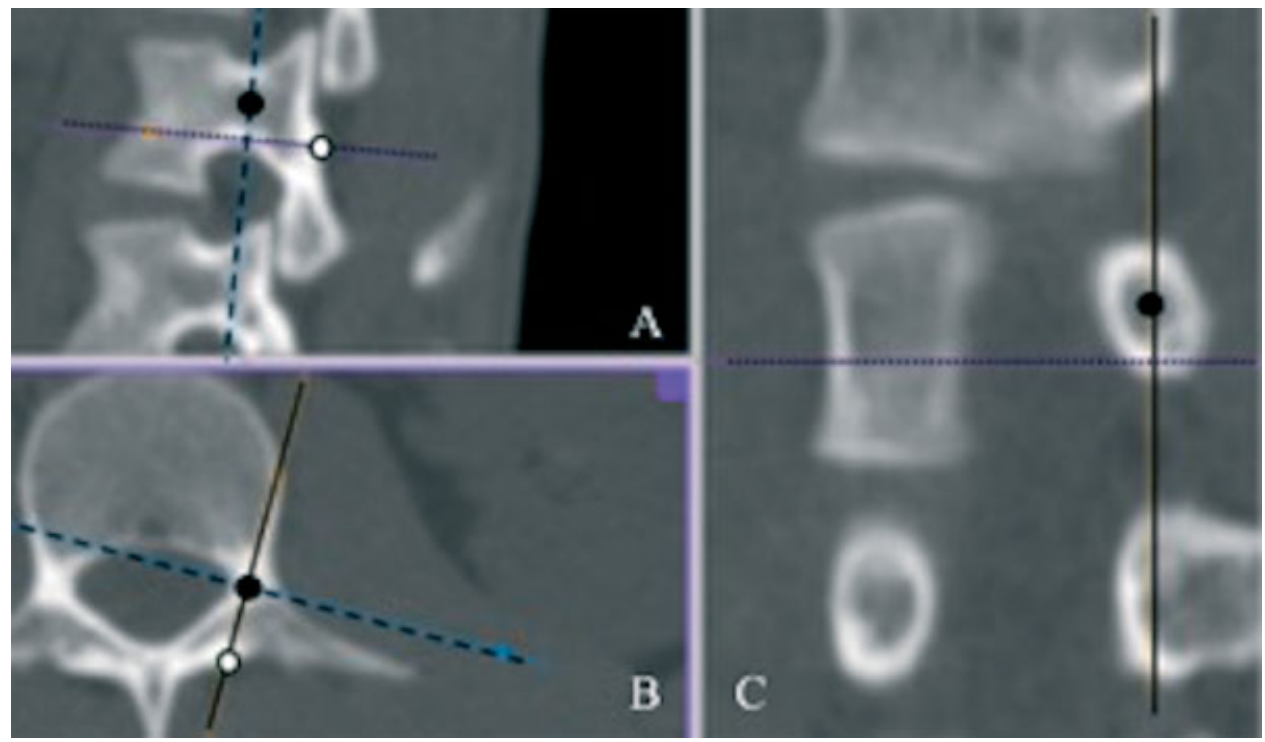

Fig. 3 Imagens sagital (A), axial (B) e coronal (C) de tomografia computadorizada demonstrando o trajeto lateral do parafuso.

encontrasse o ponto fixo 2. Assim, os cortes das imagens sagitais são gerados no eixo do trajeto do parafuso, o que permite uma correta mensuração. 0 trajeto do parafuso no plano axial foi determinado por uma linha reta que une o ponto fixo 1 (centro do eixo na imagem axial) ao ponto fixo 2 (-Figuras 3 e 4 ).

Para a medição do AL, utilizou-se a imagem axial. Determinou-se a linha sagital vertebral, uma reta formada pela união do eixo central do processo espinhoso e o ponto médio de largura do canal vertebral, e, então, mediu-se o ângulo formado pelo trajeto do parafuso e essa linha (-Figura 4).

$\mathrm{O} C \mathrm{P}$ e o AC foram mensurados no corte sagital que passa pelo eixo do parafuso. Uma linha reta entre os pontos fixos $1 \mathrm{e}$ 2 nesse corte, com início no ponto fixo 1 e término na cortical oposta, determina o máximo CP. O AC foi medido entre uma reta na placa terminal superior e o trajeto do parafuso no corte sagital inclinado (-Figura 5).

Por fim, para determinar o DP, os eixos no corte sagital foram encontrados no ponto fixo 1, e o eixo horizontal foi inclinado até se igualar ao trajeto do parafuso desenhado. $\mathrm{O}$ DP foi medido como a distância entre duas retas paralelas ao trajeto que tangenciam o córtex medial e o córtex lateral do pedículo (-Figura 6).

\section{Análise Estatística}

Foram avaliados 300 pedículos das vértebras lombares de L1 a L5. As médias e desvios padrão dos parâmetros lineares e angulares foram calculados em cada nível e para os pacientes do sexo feminino e masculino separadamente. Os valores

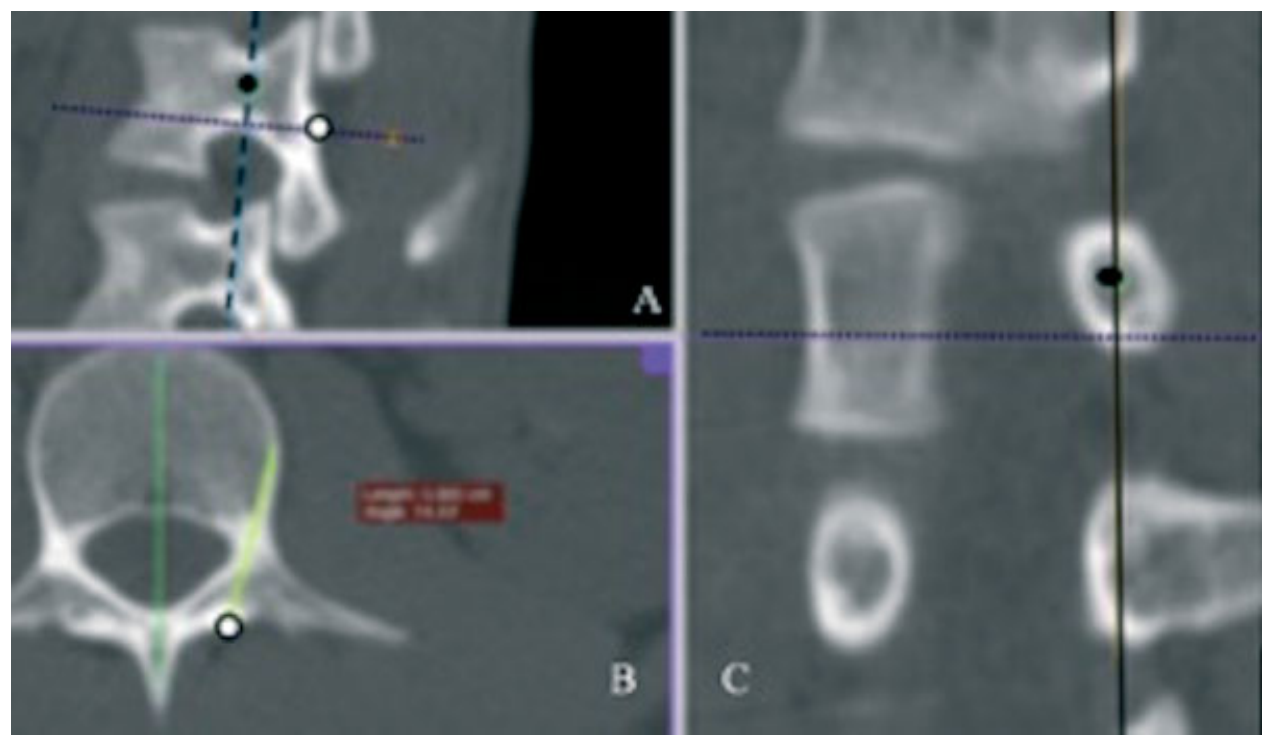

Fig. 4 Imagens sagital (A), axial (B) e coronal (C) de tomografia computadorizada evidenciando a avaliação do ângulo lateral. 


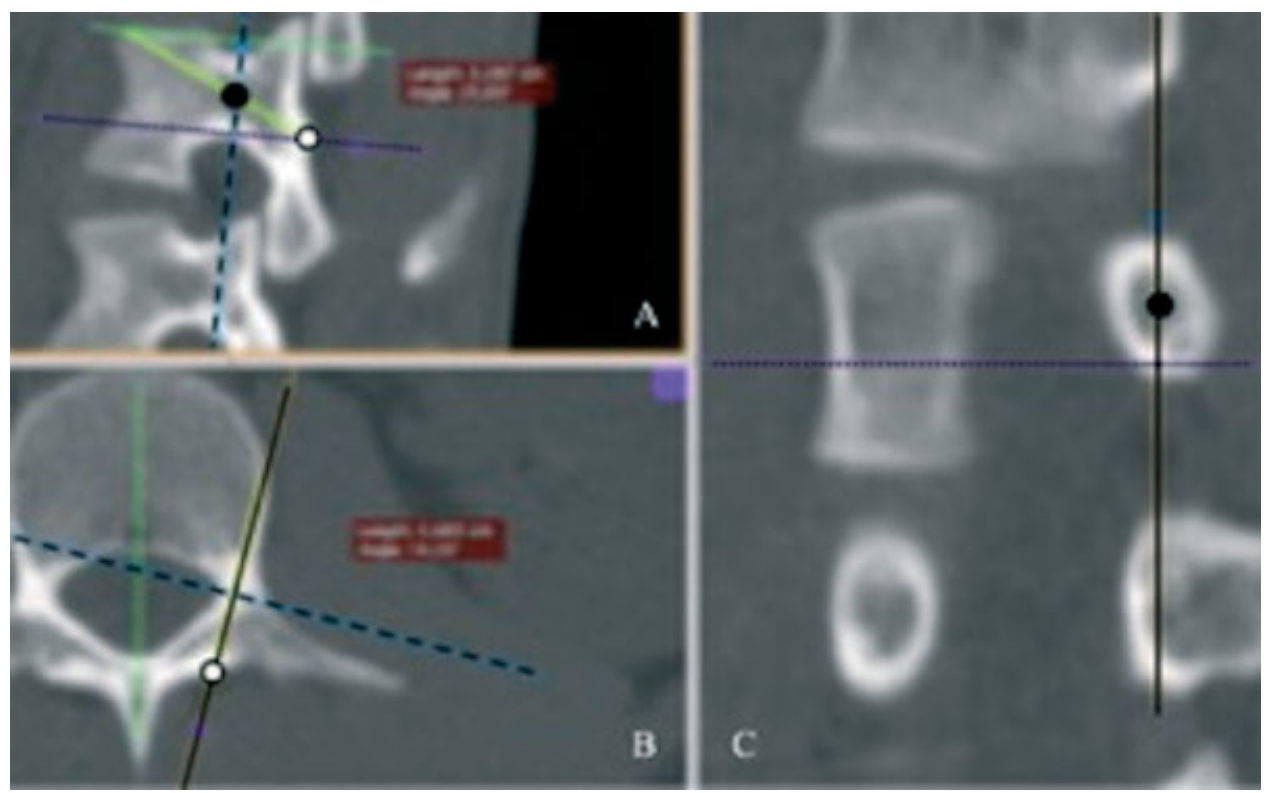

Fig. 5 Imagens sagital (A), axial (B) e coronal (C) de tomografia computadorizada demonstrando a avaliação do comprimento do trajeto do parafuso cortical e do ângulo cranial.

foram comparados com os do teste $t$ de Student após o teste de normalidade de Shapiro-Wilk.

A confiabilidade interobservador foi estimada utilizando o teste de Spearman, e foi considerada ruim para valores entre 0 e 0,21 ; fraca, entre 0,21 e 0,40; moderada, entre 0,41 e 0,60; boa, entre 0,61 e 0,80; e muito boa, entre 0,81 e 1,0. Utilizamos o nível de significância estatística $p<0,05$ para

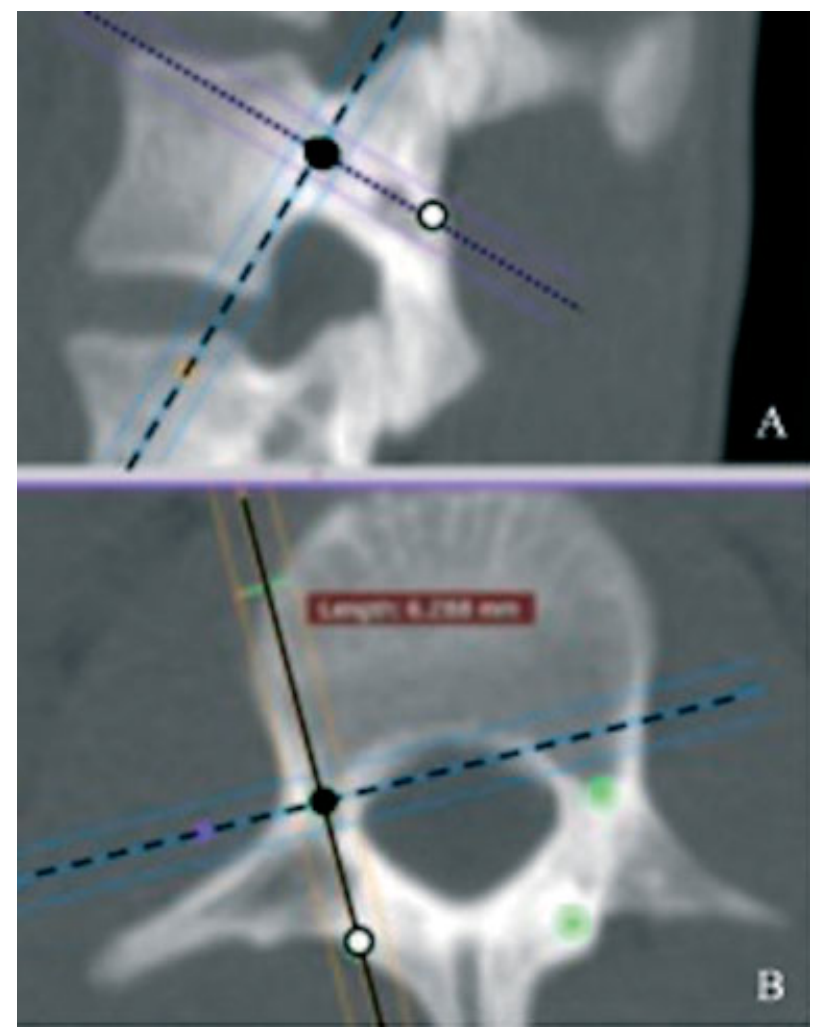

Fig. 6 Imagens sagital (A) e axial (B) de tomografia computadorizada evidenciando o cálculo do diâmetro do parafuso. todos os parâmetros, e o programa Stata (Statacorp, College Station, TX, EUA), versão 14.1, para a análise estatística.

\section{Resultados}

\section{Parâmetros Sagitais}

O CP geral variou de $19 \mathrm{~mm}$ a $45 \mathrm{~mm}$. A menor média encontrada foi no nível L5 do lado direito, com $2,65 \mathrm{~cm}$ em pacientes do sexo feminino, enquanto a maior média encontrada foi no nível L2 direito, medindo $3,36 \mathrm{~cm}$ em pacientes do sexo masculino. Houve uma tendência de diminuição nas medidas do CP conforme avançamos de proximal (L1) para distal (L5) (-Figura 7). No entanto, não encontramos diferença estatística quando comparamos os valores do $\mathrm{CP}$ entre os pedículos dos dois lados e entre os pacientes de ambos os sexos.

A média do $A C$ variou de $17,2^{\circ}$ a $25,3^{\circ}$. As menores médias foram identificadas no nível L5, e as maiores, no nível L1. Não houve diferença estatística entre os sexos ou entre os lados direto e esquerdo, com uma tendência à diminuição nos valores médios conforme avançamos de proximal (L1) para distal (L5) (-Figura 8).

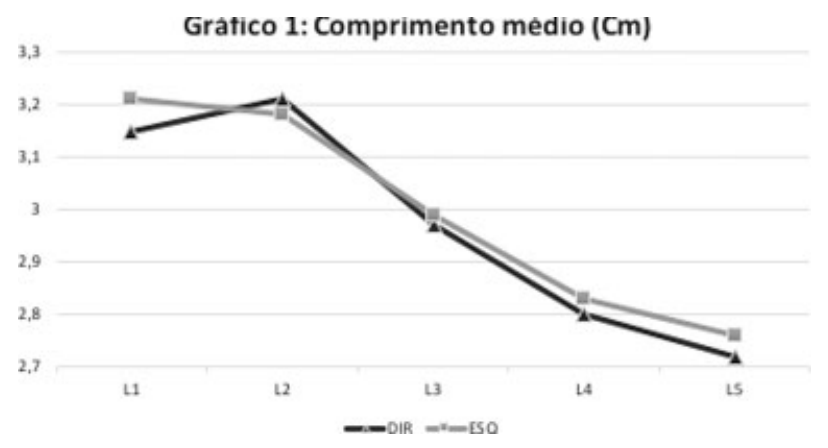

Fig. 7 Comprimento médio dos parafusos $(e m \mathrm{~cm})$ por nível. 


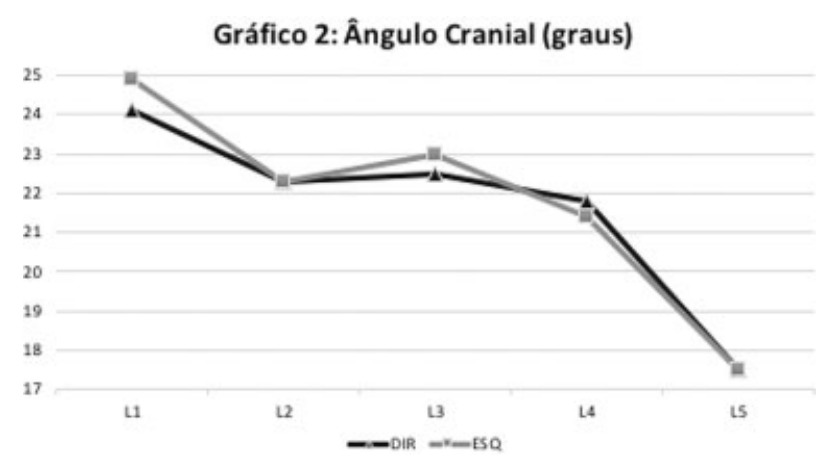

Fig. 8 Ângulo cranial médio dos parafusos (em graus) por nível.

\section{Parâmetros Axiais}

O DP geral variou de $2,3 \mathrm{~mm}$ a $7,2 \mathrm{~mm}$. A menor média encontrada foi no nível L2 do lado direito em pacientess do sexo feminino, com $4,14 \mathrm{~mm}$, enquanto a maior, no nível L5 do lado direito em pacientes do sexo masculino, com 6,1 mm. Houve uma tendência ao aumento nas medidas do DP conforme avançamos de proximal para distal (-Figura 9). Não foi encontrada diferença estatística quando comparados os valores do DP entre os dois lados ou entre os pacientes de ambos os sexos.

A média do $\mathrm{AL}$ variou de $12,8^{\circ}$ a $22,0^{\circ}$. A menor média foi identificada no nível L1 à esquerda em pacientes do sexo feminino, e a maior, no nível L4 à direita em pacientes no sexo masculino. Não houve diferença estatística entre os sexos ou os dois lados. Houve uma tendência ao aumento dos valores de proximal para distal (-Figura 10).

A tabela 1 resume os parâmetros medidos em pacientes de ambos os sexos por nível da coluna lombar.

\section{Confiabilidade Interobservador}

$\mathrm{Na}$ análise da confiabilidade interobservador por meio do teste de Spearman, não foram evidenciadas diferenças na análise de nenhum dos parâmetros, tanto sagitais quanto axiais, e a confiabilidade foi qualificada como moderada.

\section{Discussão}

O trajeto do osso cortical com o uso de PTCs é uma técnica nova de instrumentação da coluna vertebral lombar. ${ }^{3}$ Com

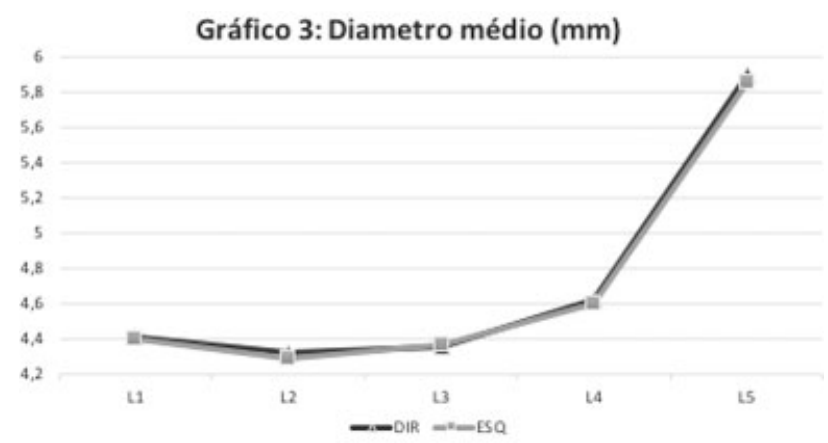

Fig. 9 Diâmetro médio dos parafusos (em mm) por nível.

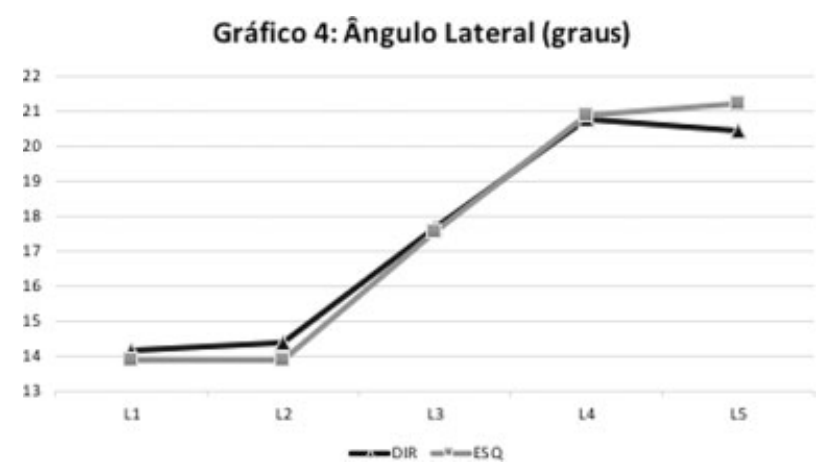

Fig. 10 Ângulo lateral médio (em graus) por nível.

seu trajeto caudal-cranial e medial-lateral, espera-se obter uma maior força de fixação, particularmente em pacientes com osso osteoporótico e idosos, pois baseia-se no maior contato do parafuso com osso cortical denso. ${ }^{4-6}$ Pelo que sabemos, esta é a primeira vez que as dimensões do trajeto do parafuso cortical em uma amostra da população brasileira são analisadas com relação ao sexo e ao nível da coluna vertebral.

Essa fixação tem se mostrado promissora, pois aumenta a força de arrancamento em $30 \%$, e a resistência dos parafusos e o torque insercional in vivo em 1,7 vezes em comparação com a fixação com PTPs. ${ }^{3,7}$ Matsukawa et al., ${ }^{8}$ em sua avaliação de elementos finitos, encontraram que os PTCs apresentam uma força de fixação por parafuso individual maior do que os PTPs, e uma rigidez adequada ao teste de flexo-extensão em uma montagem de vértebras pareadas, apesar de a montagem com os PTPs ser superior quando avaliadas a flexão lateral e a rotação axial. Por outro lado, Baluch et al. ${ }^{9}$ relataram que os PTCs têm maior resistência ao teste de perda por fadiga quando comparados com os PTPs.

Além das superioridades biomecânicas dos parafusos, a técnica exige menor dissecção de partes moles devido ao seu trajeto medial-lateral. Com uma abordagem minimamente invasiva, diminui-se a atrofia e a necrose da musculatura multífida, ${ }^{13,14}$ fato relacionado com a dor lombar persistente após a cirurgia, bem como com uma reabilitação mais precoce.

A metanálise realizada por Hu et al. ${ }^{15}$ evidenciou que a fixação com PTCs apresenta menor perda sanguínea, menor tempo de internação, menor incidência de doença do nível adjacente, e menor comprimento de incisão comparado com a fixação com PTPs. Porém, não há diferença estatísticas entre as duas técnicas com relação aos protocolos de avaliação clínicas, incidência de complicações peroperatórias, ou no tempo cirúrgico. Ainda assim, Sakaura et al. ${ }^{16}$ relataram bons resultados no seguimento de pacientes com espondilolistese tratados com artrodese por via minimamente invasiva, embora essa técnica não tenha demonstrada superioridade estatística quando comparada com a fixação com PTPs. Para pacientes obesos, a fixação com PTCs também pode ser uma técnica vantajosa devido ao profundo tecido adiposo da região lombar, o que torna desafiador a inserção dos PTPs. ${ }^{15,17}$ 
Tabela 1 Média dos parâmetros da amostra por nível

\begin{tabular}{|l|l|l|l|l|}
\hline & $\begin{array}{l}\text { Comprimento médio } \\
\text { do parafuso }(\mathrm{cm})\end{array}$ & $\begin{array}{l}\text { Diâmetro médio } \\
\text { do parafuso }(\mathrm{mm})\end{array}$ & $\begin{array}{l}\text { Ângulo lateral } \\
\text { médio }\left(^{\circ}\right)\end{array}$ & $\begin{array}{l}\text { Ângulo cranial } \\
\text { médio }\left(^{\circ}\right)\end{array}$ \\
\hline L1 & $3,18 \pm 0,55$ & $4,4 \pm 0,77$ & $14,04 \pm 3,28$ & $24,53 \pm 3,59$ \\
\hline L2 & $3,19 \pm 0,37$ & $4,30 \pm 0,68$ & $14,13 \pm 2,88$ & $22,54 \pm 3,16$ \\
\hline L3 & $2,98 \pm 0,46$ & $4,36 \pm 0,81$ & $17,64 \pm 3,25$ & $22,76 \pm 3,34$ \\
\hline L4 & $2,81 \pm 0,38$ & $4,61 \pm 0,56$ & $20,84 \pm 3,76$ & $21,6 \pm 3,47$ \\
\hline L5 & $2,74 \pm 0,29$ & $5,88 \pm 0,64$ & $20,82 \pm 3,98$ & $17,52 \pm 3,0$ \\
\hline
\end{tabular}

O PTC provou-se ser vantajoso em evitar a violação da cortical medial ou lateral do pedículo quando comparado ao PTP, preservando, assim, a integridade das estruturas neurais, embora complicações tenham sido relatadas, principalmente no início da curva de aprendizado da técnica. ${ }^{18}$ Outras complicações potenciais são a soltura precoce dos implantes, infecção, fratura da pars interarticularis ou do pedículo, e lesão da raiz nervosa. Uma desvantagem do PTC consiste na limitação de realizar montagens híbridas devido ao fato de as cabeças dos parafusos não estarem alinhadas com os PTPs, dificultando a colocação da haste. ${ }^{18,19}$

Embora essa técnica já tenha ganhado certa popularidade nos últimos anos, existem poucos estudos que avaliam a morfologia do trajeto e o tamanho dos implantes adequados para diferentes populações visando uma cirurgia mais segura com menor taxa de complicações. ${ }^{10-12}$ O primeiro grande estudo com analise morfométrica por meio de exame de TC foi realizado por Matsukawa et al. ${ }^{10}$ em uma amostra da população japonesa, que ajudou a desenvolver a técnica hoje utilizada. Posteriormente, Zhang et al. ${ }^{11}$ e Senoglu et al. ${ }^{12}$ também realizaram seus estudos anatômicos com base em exames de TC na China e na Turquia, respectivamente. Embora os resultados sejam similares, apresentam diferenças importantes, que podem levar a complicações quando não levados em conta ao se realizar um procedimento cirúrgico.

Matsukawa et al. ${ }^{10}$ encontraram um CP médio de $3,68 \mathrm{~cm}$ a $3,98 \mathrm{~cm}$; Zhan et al., ${ }^{11}$ uma média de $2,79 \mathrm{~cm}$ a $2,9 \mathrm{~cm}$; Senoglu et al., ${ }^{12}$ de $2,70 \mathrm{~cm}$ a $4,0 \mathrm{~cm}$, a depender do padrão de medição utilizado; em nosso estudo, encontramos valores médios de $2,72 \mathrm{~cm}$ a $3,11 \mathrm{~cm}$, ou seja, semelhante aos dos estudos prévios. Quanto ao DP, observamos uma grande variabilidade nos tamanhos: Matsukawa et al. ${ }^{10}$ apresentaram valores entre $6,2 \mathrm{~mm}$ e $8,4 \mathrm{~mm}$; Zhang et al., ${ }^{11}$ entre 7,8 mm e 10,4 mm; Senoglu et al., ${ }^{12}$ entre 4,5 mm e 5,0 mm; e nossos resultados variaram entre $4,3 \mathrm{~mm}$ e $5,9 \mathrm{~mm}$. O AC também apresentou diferenças entre os estudos, uma vez que Matsukawa et al. ${ }^{10}$ encontraram ângulos variando entre $25,5^{\circ}$ e $26,2^{\circ}$; Zhang et al., ${ }^{11}$ entre $22,9^{\circ}$ e $26,7^{\circ}$; Senoglu et al., ${ }^{12}$ entre $31^{\circ}$ e $48^{\circ}$; e nossas medidas variaram entre $17,5^{\circ}$ e $24,9^{\circ}$.

Tais diferenças podem ser justificadas tanto pela heterogeneidade das populações estudadas quanto pelo o predomínio de homens no primeiro estudo ${ }^{10}$ (90\%), que foi realizado em uma população militar, assim como pelos diferentes protocolos de medição descritos pelos três estudos anteriores ${ }^{10-12}$. As medições nos estudos prévios foram realizadas com os planos sagital e axial em posição anatômica; porém, o parafuso não é inserido no eixo anatômico da vértebra, mas inclinado para lateral. Assim, essa forma de medição pode falsear o resultado, principalmente quanto ao $\mathrm{CP}$.

A descrição da técnica de medição do presente estudo leva em consideração o trajeto ideal do parafuso, alterando o eixo do corte sagital para coincidir com o trajeto do parafuso; assim, conseguimos obter a medida exata dos parâmetros.

Parafusos com comprimento e diâmetro aumentados podem violar as corticais lateral e medial do pedículo e colocar em risco estruturas nervosas, bem como levar a fratura da pars e invasão do disco vertebral. ${ }^{18}$ Portanto, consideramos fundamental a avaliação radiológica prévia ao procedimento cirúrgico envolvendo a utilização do PTC.

Embora nossos resultados não apresentem diferença estatística entre os sexos, observa-se uma média maior nos parâmetros avaliados em pacientes do sexo masculino, fato que pode justificar-se pelo tamanho relativamente pequeno da amostra. Tal restrição na amostra é uma limitação do presente estudo; porém, este é o primeiro estudo a englobar uma amostra da população brasileira, com alto nível de miscigenação, e a descrever uma técnica pormenorizada da exata mensuração das dimensões dos parafusos, respeitando o eixo do parafuso e o AL e AC.

Nosso método de mensuração se mostrou eficaz, com boa reprodutibilidade interobservador, mas um estudo subsequente para a validação das medidas comparando os dados digitais com as medições realizadas no intraoperatório se faz necessário.

\section{Conclusão}

O parafuso de trajeto do osso cortical apresenta uma variação em suas características morfométricas relacionadas com o trajeto anatômico de inserção, quando avaliamos as diferentes vértebras lombares, não apresentando na amostra estudada diferença estatística entre os sexos. Assim, recomendamos um estudo pré-operatório de imagens detalhado para reduzir os riscos cirúrgicos relacionados à técnica. 


\section{Suporte Financeiro}

Não houve suporte financeiro de fontes públicas, comerciais, ou sem fins lucrativos.

\section{Conflito de Interesses}

Os autores declaram não haver conflito de interesses.

\section{Referências}

1 Cook SD, Barbera J, Rubi M, Salkeld SL, Whitecloud TS 3rd. Lumbosacral fixation using expandable pedicle screws. an alternative in reoperation and osteoporosis. Spine J 2001;1(02):109-114

2 Mobbs RJ. The "medio-latero-superior trajectory technique": an alternative cortical trajectory for pedicle fixation. Orthop Surg 2013;5(01):56-59

3 Phan K, Ramachandran V, Tran TM, et al. Systematic review of cortical bone trajectory versus pedicle screw techniques for lumbosacral spine fusion. J Spine Surg 2017;3(04):679-688

4 Kojima K, Asamoto S, Kobayashi Y, Ishikawa M, Fukui Y. Cortical bone trajectory and traditional trajectory-a radiological evaluation of screw-bone contact. Acta Neurochir(Wien) 2015;157(07):1173-1178

5 Mai HT, Mitchell SM, Hashmi SZ, Jenkins TJ, Patel AA, Hsu WK. Differences in bone mineral density of fixation points between lumbar cortical and traditional pedicle screws. Spine J 2016;16(07):835-841

6 Zhang R, Gao H, Li H, et al. Differences in bone mineral density of trajectory between lumbar cortical and traditional pedicle screws. J Orthop Surg Res 2019;14(01):128-135

7 Matsukawa K, Yato Y, KatoT, Imabayashi H, Asazuma T, Nemoto K. In vivo analysis of insertional torque during pedicle screwing using cortical bone trajectory technique. Spine (Phila Pa 1976) 2014;39 (04):E240-E245

8 Matsukawa K, Yato Y, Imabayashi H, Hosogane N, Asazuma T, Nemoto K. Biomechanical evaluation of the fixation strength of lumbar pedicle screws using cortical bone trajectory: a finite element study. J Neurosurg Spine 2015;23(04):471-478

9 Baluch DA, Patel AA, Lullo B, et al. Effect of physiological loads on cortical and traditional pedicle screw fixation. Spine (Phila Pa 1976) 2014;39(22):E1297-E1302
10 Matsukawa K, Yato Y, Nemoto O, Imabayashi H, Asazuma T, Nemoto K. Morphometric measurement of cortical bone trajectory for lumbar pedicle screw insertion using computed tomography. J Spinal Disord Tech 2013;26(06):E248-E253

11 Zhang H, Ajiboye RM, Shamie AN, Wu Q Chen Q, Chen W. Morphometric measurement of the lumbosacral spine for minimally invasive cortical bone trajectory implant using computed tomography. Eur Spine J 2016;25(03):870-876

12 Senoglu M, Karadag A, Kinali B, Bozkurt B, Middlebrooks EH, Grande AW. Cortical Bone Trajectory Screw for Lumbar Fixation: A Quantitative Anatomic and Morphometric Evaluation. World Neurosurg 2017;103:694-701

13 Hung CW, Wu MF, Hong RT, Weng MJ, Yu GF, Kao CH. Comparison of multifidus muscle atrophy after posterior lumbar interbody fusion with conventional and cortical bone trajectory. Clin Neurol Neurosurg 2016;145:41-45

14 Chen YR, Deb S, Pham L, Singh H. Minimally Invasive Lumbar Pedicle Screw Fixation Using Cortical Bone Trajectory - A Prospective Cohort Study on Postoperative Pain Outcomes. Cureus 2016;8(07):e714-e726

15 Hu JN, Yang XF, Li CM, Li XX, Ding YZ. Comparison of cortical bone trajectory versus pedicle screw techniques in lumbar fusion surgery: A meta-analysis. Medicine (Baltimore) 2019;98(33): e16751-e16758

16 Sakaura H, Miwa T, Yamashita T, Kuroda Y, Ohwada T. Cortical bone trajectory screw fixation versus traditional pedicle screw fixation for 2-level posterior lumbar interbody fusion: comparison of surgical outcomes for 2-level degenerative lumbar spondylolisthesis. J Neurosurg Spine 2018;28(01):57-62

17 Santoni BG, Hynes RA, McGilvray KC, et al. Cortical bone trajectory for lumbar pedicle screws. Spine J 2009;9(05):366-373

18 Dayani F, Chen YR, Johnson E, et al. Minimally invasive lumbar pedicle screw fixation using cortical bone trajectory - Screw accuracy, complications, and learning curve in 100 screw placements. J Clin Neurosci 2019;61:106-111

19 Patel SS, Cheng WK, Danisa OA. Early complications after instrumentation of the lumbar spine using cortical bone trajectory technique. J Clin Neurosci 2016;24:63-67 